\section{Secondary-care costs associated with lung cancer diagnosed at emergency hospitalisation in the United Kingdom}

\begin{abstract}
Lung cancer diagnosis during emergency hospital admission has been associated with higher early secondary-care costs and lower longer-term costs than outpatient diagnoses. This retrospective cohort study analyses the secondary-care costs of 3274 consecutive patients with lung cancer. Patients diagnosed during emergency admissions incurred greater costs during the first month and had a worse prognosis compared with outpatient diagnoses. In patients who remained alive, costs after the first month were comparable between diagnostic routes. In addition to improving patient experience and outcome, strategies to increase earlier diagnosis may reduce the additional healthcare costs associated with this route to diagnosis.
\end{abstract}

\section{INTRODUCTION}

In the UK, $35 \%$ of new lung cancers are diagnosed during an emergency presentation. ${ }^{1}$ This is associated with poor outcomes. ${ }^{2}$ Hospital admissions are a poor patient experience. ${ }^{3}$ Reducing the proportion of patients diagnosed by this route has been identified as a priority by policy makers.

Inpatient admissions are the greatest contributor to secondary-care costs in patients with lung cancer. ${ }^{4-7}$ Only one small study has previously examined costs by route to diagnosis. ${ }^{5}$ This identified that early costs were higher in patients diagnosed during an acute admission than those diagnosed following outpatient referral. Our data also previously identified that diagnosis during an acute hospital admission was associated with higher 90-day costs, although costs were lower at 1 year. We hypothesised that this was due to the excess mortality in this poor prognosis group. ${ }^{7}$

The full health-economic impact of this route to diagnosis is not known. The aim of this study was to further analyse the effect of lung cancer diagnosis during emergency admission on secondary-care costs.

\section{METHODS}

This was a retrospective cohort study. All patients first diagnosed with lung cancer at Leeds Teaching Hospitals NHS Trust between January 2008 and October 2013 were included.

Costs were based on the recorded hospital income, based on human resource group coding and national payment by results tariff. This represents the cost to the local National Health Service (NHS) commissioners for inpatient, outpatient and emergency department care. Community and palliative care costs were not available. All costs were adjusted to 2013-2014 prices. Day zero was defined as the date first seen by the lung cancer team.

Patients diagnosed during an emergency admission were compared with those diagnosed in outpatient clinics. Patient characteristics were compared using t-test (age) and $\chi^{2}$ analyses (all other characteristics). Survival was compared using Cox proportional hazard analysis. Ordinary least squares regression analyses of both 90-day and 1-year log-costs were performed. Covariates included in each analysis were age, performance status, route to diagnosis, diagnosis year, stage, histology, epidermal growth factor receptor (EGFR) gene mutation status and treatment received. Monthly costs were calculated for each patient.

\section{RESULTS}

There were 3289 consecutively diagnosed patients. After exclusion of 15 patients (13 second or recurrent lung cancers and 2 corrupted data), 3274 patients were included for analysis. All patients had a minimum of 1 year follow-up.

There were 1027 (31.4\%) patients diagnosed during an emergency admission and $2247(68.6 \%)$ as outpatients.

Compared with outpatients, patients diagnosed during emergency admissions were older (mean age 73.9 vs 71.9 years, $\mathrm{p}<0.001$ ), with a higher proportion with performance status 3-4 (61.7\% vs $24.4 \%$, $\mathrm{p}<0.001)$ and stage IIIB-IV disease $(80.1 \%$ vs $50.4 \%, \mathrm{p}<0.001)$. They were less likely to have a histologically confirmed diagnosis $(54.1 \%$ vs $78.2 \%$, $\mathrm{p}<0.001)$ or to receive radical treatment $(5.8 \%$ vs $35.4 \%, \mathrm{p}<0.001)$ and more likely to receive best supportive care $(62.7 \%$ vs $25.7 \%, \mathrm{p}<0.001)$. One-year survival was significantly worse $(13.7 \%$ (95\% CI $11.7 \%$ to $15.9 \%)$ vs $50.2 \%$ (95\% CI $48.2 \%$ to $52.3 \%)$, HR 0.294 (95\% CI 0.268 to 0.321$), \mathrm{p}<0.001)$. Median length of stay for those diagnosed during emergency admission was 12 days (IQR 622 days). The mean cost per bed-day during these initial admissions was $£ 203$.

The mean 1-month and 1-year costs for all cases were $£ 2400$ (95\% CI $£ 2313$ to
$£ 2493$ ) and $£ 10009$ (95\% CI $£ 9725$ to $£ 10285)$, respectively.

Univariate regression analysis shows that diagnosis during emergency admission is associated with increased 90-day $(p=0.013)$ and reduced 1-year $(p<0.001)$ costs. Multivariate regression, however, shows this to increase both costs $(\mathrm{p}<0.001)$.

Figure 1 shows the mean monthly costs for all patients by route to diagnosis. Compared with outpatient diagnoses, diagnosis during emergency admission was associated with higher costs over the first month ( $£ 3499$ (95\% CI $£ 3332$ to $£ 3667)$ vs $£ 1899$ (95\% CI $£ 1810$ to $£ 1999)$ ), but lower costs over 1 year (£8123 (95\% CI $£ 7704$ to $£ 8552)$ vs $£ 10870$ (95\% CI $£ 10511$ to $£ 11211$ )). This is likely to have been heavily influenced by excess mortality in this group.

Figure 2 shows the costs adjusted for survival, considering mean costs per patient alive in that month. Emergency admission was associated with increased cumulative mean alive costs over 1 year compared with outpatients ( $£ 15059$ (95\% CI $£ 14235$ to $£ 15966)$ vs $£ 13221$ (95\% CI $£ 12885$ to $£ 13580)$ ). The excess mean alive cost accumulated over the first year after diagnosis during emergency admission was $£ 1838$. Cumulative mean alive costs accrued between months 2 and 12 were similar between the two groups ( $£ 11577$ (95\% CI $£ 10836$ to $£ 12392$ ) vs $£ 11321$ (95\% CI $£ 10979$ to $£ 11$ 663)).

The proportion of cumulative 1 year costs that were attributable to inpatient and emergency department costs was greater for those diagnosed during an emergency admission (60.7\% vs $35.4 \%$ ).

\section{DISCUSSION}

This is the largest analysis of direct hospital costs in the management of lung cancer according to route to diagnosis. This study used hospital income to reflect costs, which provides an electronic source of individualised costs that represent the true cost to commissioners. This may differ from the true cost to the hospital.

Emergency presentation was associated with a number of adverse parameters (worse performance status, more advanced disease, less radical treatment, shorter survival). These patients incur higher healthcare costs during the first month after diagnosis. Their low rate of histological confirmation and radical treatment, as well as the increased proportion of costs due to inpatient activity, suggest that this is due to the cost of the emergency admission rather than differing early investigations or cancer 


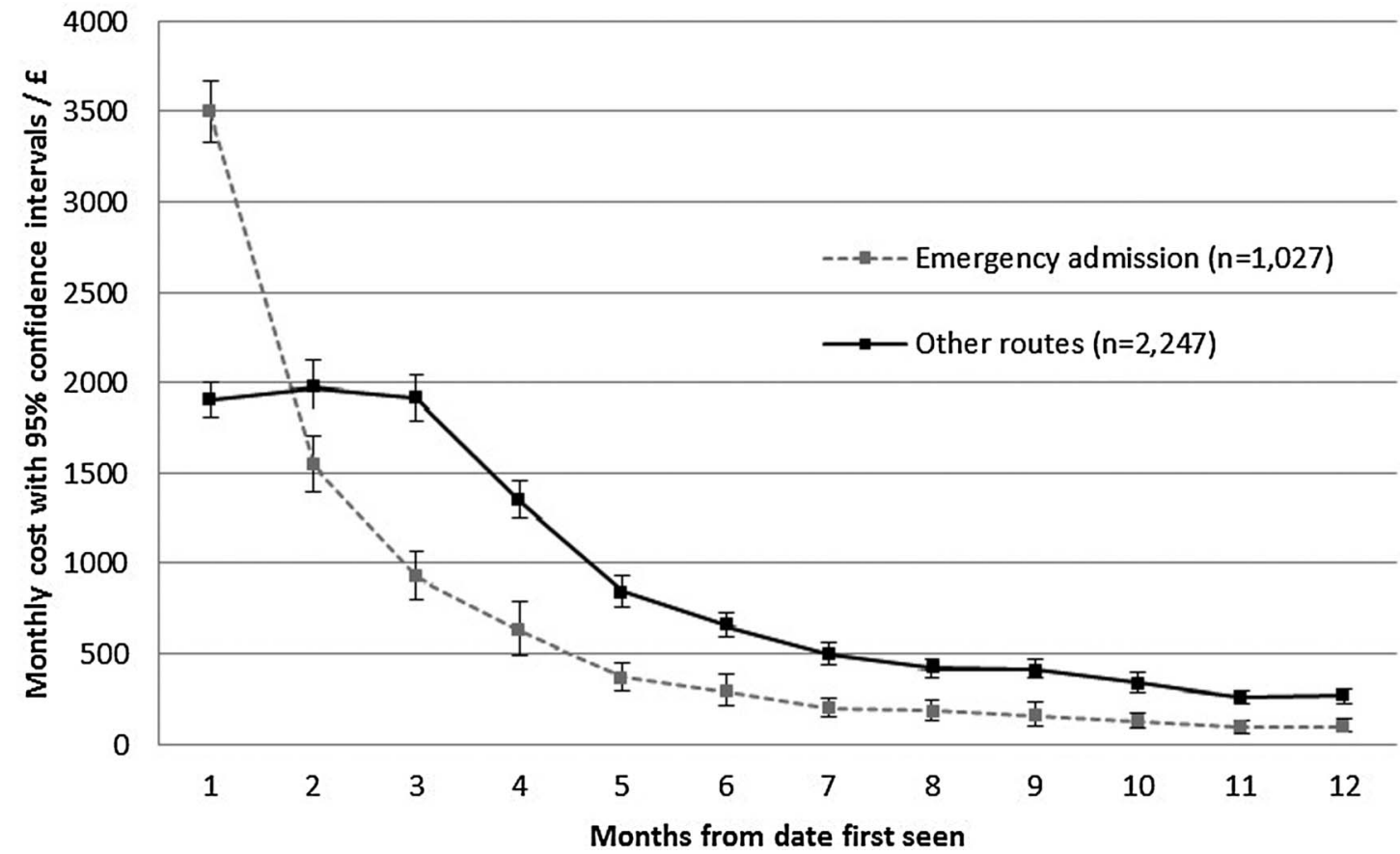

Figure 1 Mean monthly costs.

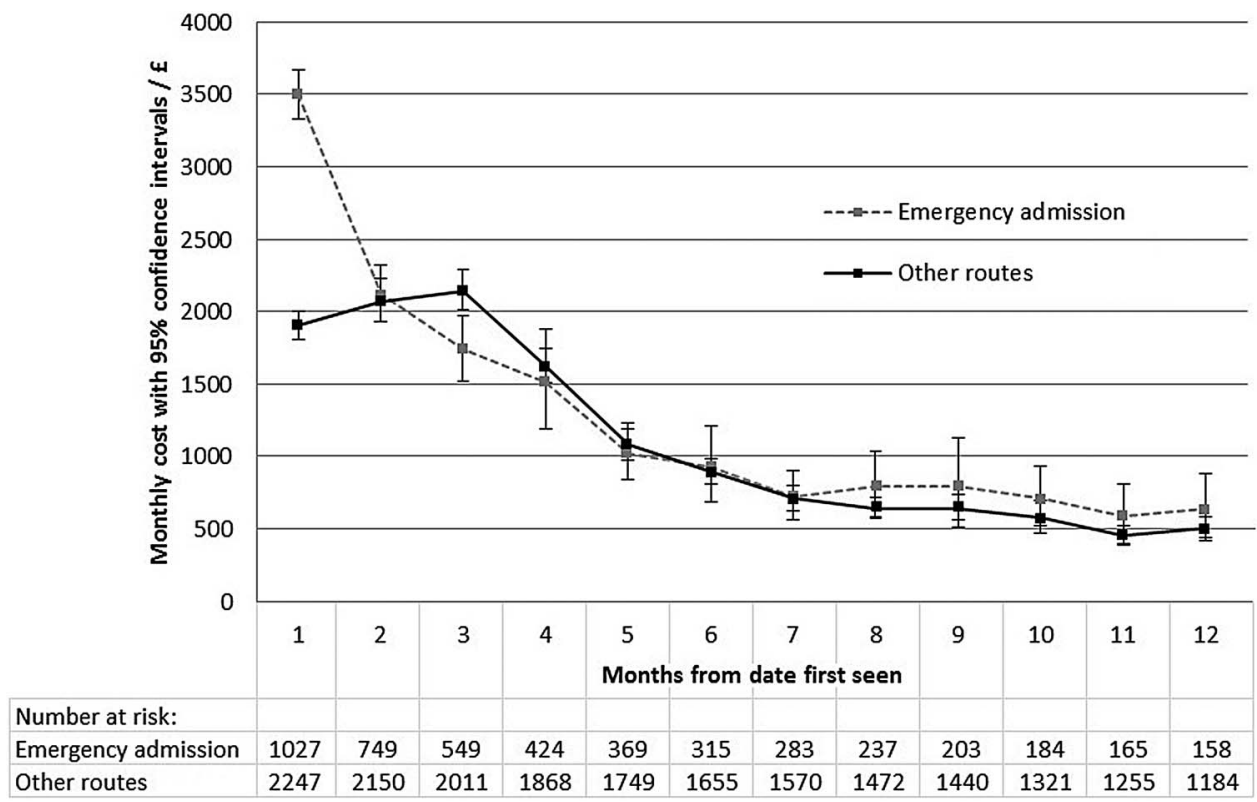

Figure 2 Mean monthly cost of care while alive (censored for survival).

treatment. Lower costs after this time probably reflect excess mortality. In patients who remain alive, costs after the first month are comparable to outpatient diagnoses and the excess cost in the first month is the only measurable cost difference. Other studies have identified high hospital costs associated with the terminal phase of cancer care. ${ }^{8}$ A limitation of this study is not having access to primary-care, socialcare and palliative-care costs, which are a significant contributor to cancer costs. ${ }^{9} 10$
The excess cost of diagnosis through emergency admission in this study was $\sim £ 1800$ per patient over the year after diagnosis. Healthcare systems with a higher cost per inpatient bed-day may find higher excess costs. In addition to improving patient experience and outcome, strategies to increase earlier diagnosis of lung cancer and reduce emergency diagnoses may reduce the additional healthcare costs associated with this route to diagnosis.
Martyn P T Kennedy, ${ }^{1}$ Peter S Hall, ${ }^{2}$ Matthew E J Callister ${ }^{1}$

1 Leeds Teaching Hospitals NHS Trust, Leeds, UK ${ }^{2}$ Edinburgh Cancer Research Centre, University of Edinburgh, Edinburgh, UK

Correspondence to Dr Martyn P T Kennedy, Leeds Teaching Hospitals NHS Trust, c/o Dr M Callister, Level 4, Gledhow Wing, Beckett Street, Leeds LS9 7TF, UK; martyn.kennedy@nhs.net

Contributors All authors contributed to the data analysis, manuscript preparation and manuscript review. The final research letter has been approved by all 
authors. The revised research letter has been approved by all authors.

Competing interests None declared.

Provenance and peer review Not commissioned; externally peer reviewed.

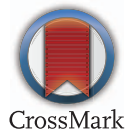

To cite Kennedy MPT, Hall PS, Callister MEJ. Thorax 2017;72:950-952.

Received 21 October 2016

Revised 10 January 2017

Accepted 13 January 2017

Published Online First 30 January 2017

Thorax 2017:72:950-952.

doi:10.1136/thoraxjnl-2016-209616

\section{REFERENCES}

1 National Cancer Intelligence Network. Routes to diagnosis. London: Public Health England, 2014. http://www.ncin.org.uk/publications/routes_to_ diagnosis (accessed Feb 2016).

2 McPhail S, Elliss-Brookes L, Shelton J, et al. Emergency presentation of cancer and short-term mortality. Br J Cancer 2013;109:2027-34.

3 King J, Ingham-Clark C, Parker C, et al. Towards saving a million bed days: reducing length of stay through an acute oncology model of care for inpatients diagnosed as having cancer. BMJ Qual Saf 2011;20:718-24

4 Fleming I, Monaghan P, Gavin A, et al. Factors influencing hospital costs of lung cancer patients in Northern Ireland. Eur I Health Econ 2008;9:79-86

5 Arca JA, Ramos MA, de la Infanta RG, et al. Lung cancer diagnosis: hospitalization costs. Arch Bronconeumol 2006;42:569-74.
6 Kang S, Koh ES, Vinod SK, et al. Cost analysis of lung cancer management in South Western Sydney. I Med Imaging Radiat Oncol 2012;56 235-41.

7 Kennedy MPT, Hall PS, Callister MEJ. Factors affecting hospital costs in lung cancer patients in the United Kingdom. Lung Cancer 2016;97:8-14.

8 Laudicella M, Walsh M, Burns E, et al. Cost of care for cancer patients in England: evidence from population-based patient-level data. $\mathrm{Br} J$ Cancer 2016;114:1286-92.

9 Chitnis X, Steventon A, Glaser A, et al. Use of health and social care by people with cancer. London: Nuffield Trust, 2014. http://www.nuffieldtrust.org.uk/ publications/use-health-and-social-care-people-cancer (accessed 22 Mar 2016).

10 Khan NF, Watson E, Rose PW. Primary care consultation behaviours of long-term, adult survivors of cancer in the UK. Br J Gen Pract 2011;61:197-9. 